\title{
Step Pyramid Distribution for Prime Numbers
}

\author{
Shaimaa said $\operatorname{soltan}^{1}$ \\ ${ }^{1}$ Computer Engineer, Toronto, Canada \\ Correspondence: Shaimaa Soltan, 3050 Constitution Blvd, Mississauga, ON., L4Y 3X1, Canada
}

Received: November 20, 2021 Accepted: January 4, 2022 Online Published: January 14, 2022

doi:10.5539/jmr.v14n1p55

URL: https://doi.org/10.5539/jmr.v14n1p55

\begin{abstract}
In this document, we will present a new way to visualize the distribution of Prime Numbers in the number system to spot Prime numbers in a subset of numbers using a simpler algorithm.

Then we will look throw a classification algorithm to check if a number is prime using only 7 simple arithmetic operations with an algorithm complexity less than or equal to $\mathrm{O}(7)$ operations for any number.
\end{abstract}

Keywords: prime numbers, composite prime numbers, prime number distribution

\section{Introduction}

\subsection{Introduce the Problem}

Prime numbers distribution is not clear and is a missing part of the number system theory.

So, we need to find a clear visual way that helps in revealing a clear distribution for the prime numbers.

In Figure 1., we define a new graphical unit to explain how this distribution works

B is the distribution rotation starting point.

Base Point of Pyramid Level /Step.

Natural Number divisible by 3.

In First Level in $\mathrm{N}$ numbers pyramid, B will be

Least multiplier of 3 in a subset of $\mathrm{N}$ number.

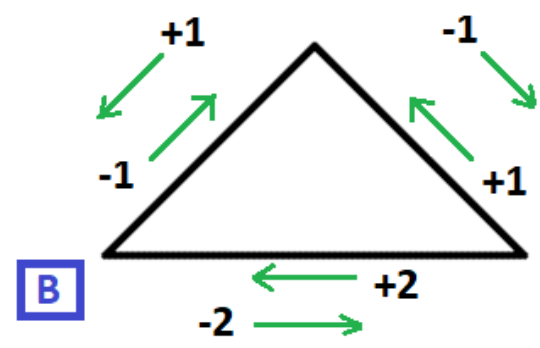

Figure 1. Pyramid Triangle Unit

We will use this unit triangle as our building block in this pyramid distribution and each vertex on this triangle will be used to position the numbers. One of these three vertices will be used as our base for the rotation and calculations.

This base vertex will be in a specific location on the triangle (left bottom vertex). Any number located on this vertex we will call it Level Base number.

For Example, for number 1,2,3 the triangle will be

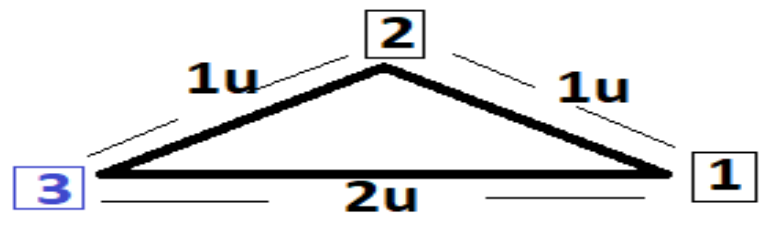

Figure 2. Pyramid Triangle Unit for numbers from 1 to 3 
next, we will see how the other numbers will continue to rotate around this unit triangle with a clear distribution based on the level of rotation and the position of the numbers related to the base number.

Let us start with the natural numbers from 1 to 15 for simplicity, using our unit triangle and its operations.

1- The Base is on the bottom left vertex.

2- The number on the vertex on the right of the base vertex will be equal to the base number -2 .

3- The number on the vertex on the top of the base vertex will be the (base number -1).

There is a specific long proof for this distribution but not included in detail in this paper, we will just describe the final shape of the distribution for these numbers

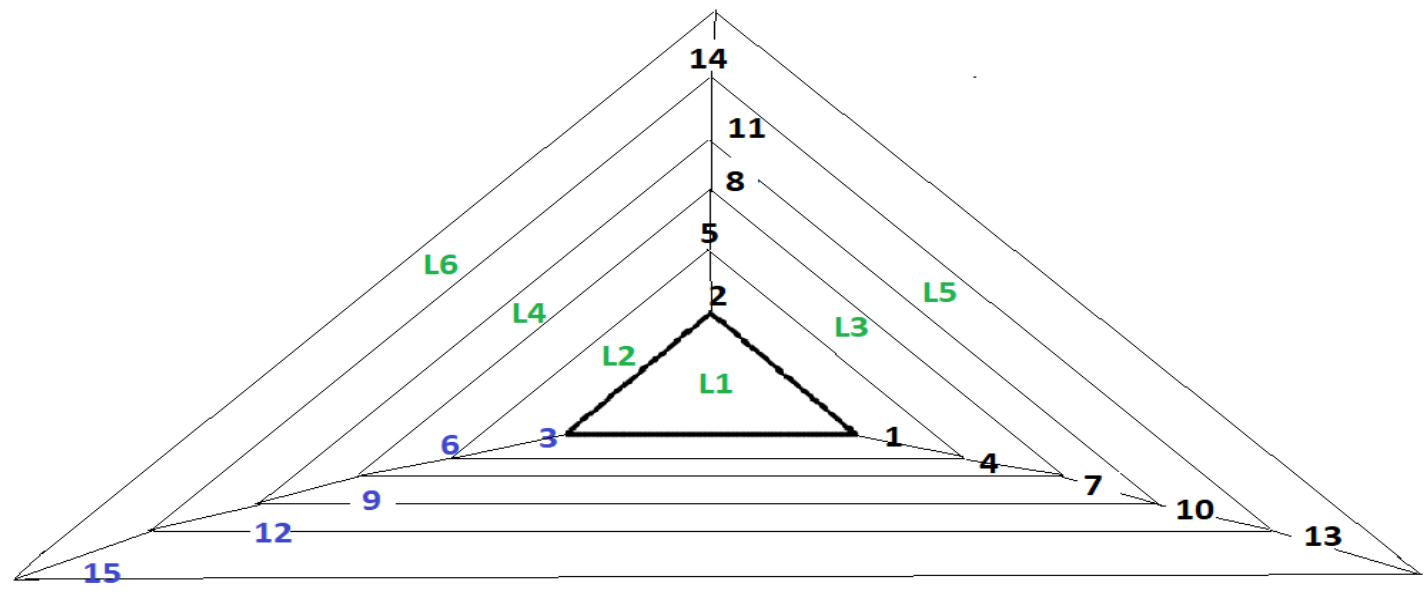

Figure 3. Pyramid Distribution for numbers 1 to 15

From this distribution, there are three main points to keep:

1- The Left bottom branch (Blue Numbers) is all the natural numbers that are divisible by 3 .

2- Each triangle will be a new level in the pyramid, represented by green letters L1, L2, L3, L4, ..

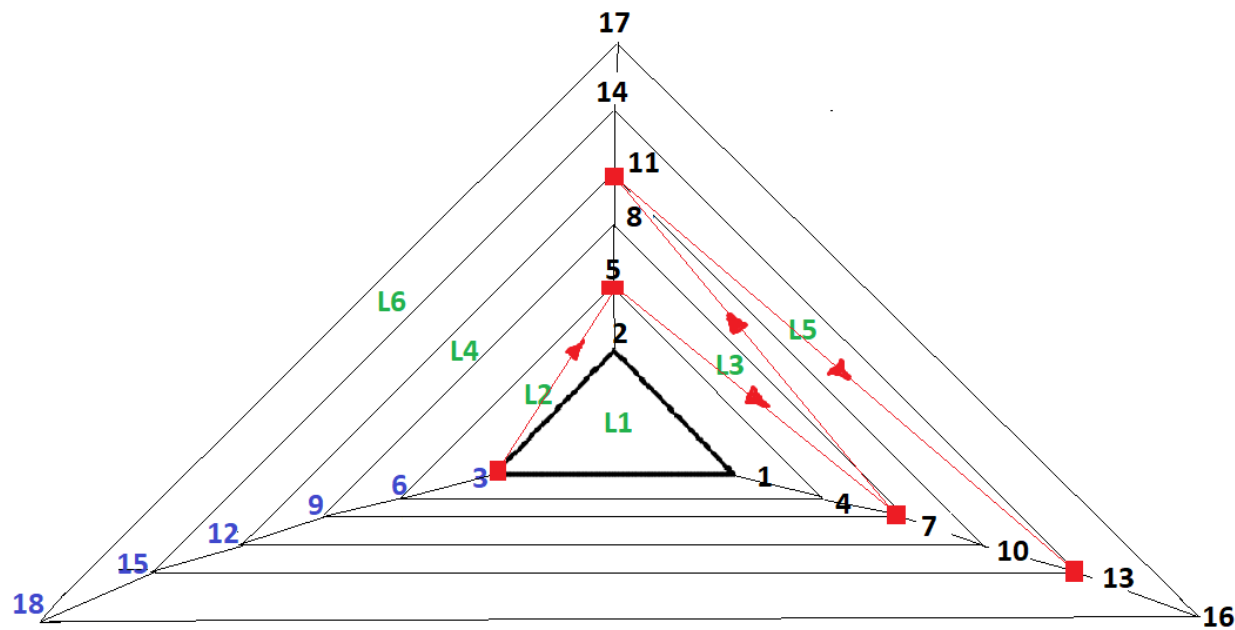

Figure 4. Pyramid Distribution for Prime Numbers Between Levels

3- Except for number 3 all the Prime numbers and composite primes will be positioned on the other two vertices. (Number 3 and its multipliers will be on Base vertex [B]) 
4- These prime numbers will be jumping between these two vertices moving one on level up as shown in (Figure 4)

i.e., Starting from Level 2 (L2), the first Prime (Number 5) is positioned on a top vertex in the level 2 triangle. So, the next prime will be in level 3 (L3) on the right vertex (Number 7), and the next prime will be in level 4 (L4) on the top vertex (number 11), and so on.

Based on this distribution you can rebuild any pyramid with the same concept without the need to build the full levels for the pyramid as in the following graph (Figure 5), where some levels are skipped in the graph. But the unit triangle properties still hold

1- Base vertices have all the natural numbers divisible by 3 (Numbers in Blue)

2- All prime numbers will be on either the top vertex or in the right vertex from the base vertex.

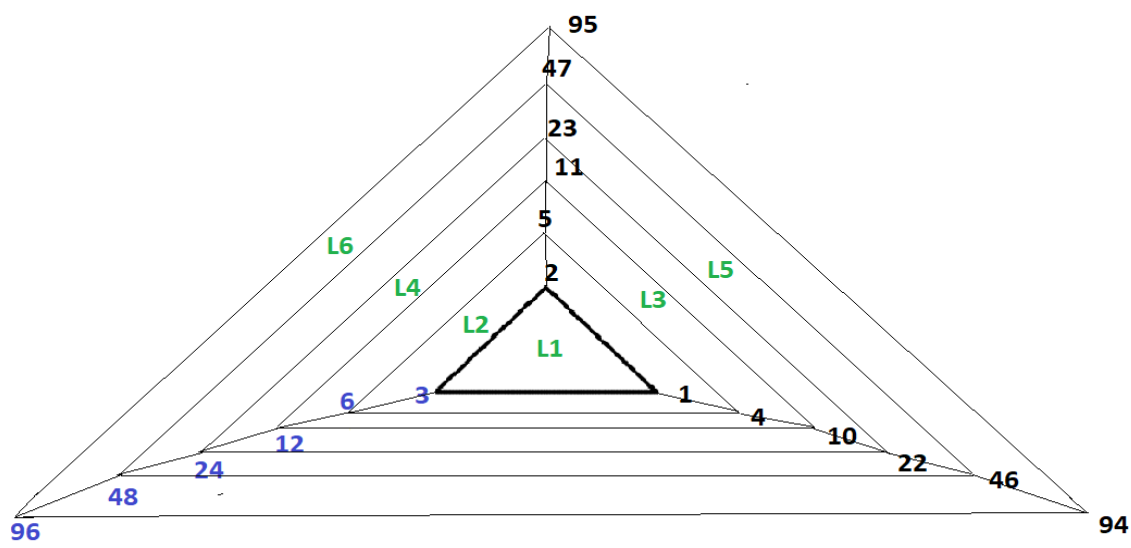

Figure 5. Sub Pyramid Distribution skip Some Levels

For example, if we built the pyramid for the natural numbers between 31 and 45 the unit triangle properties will hold.

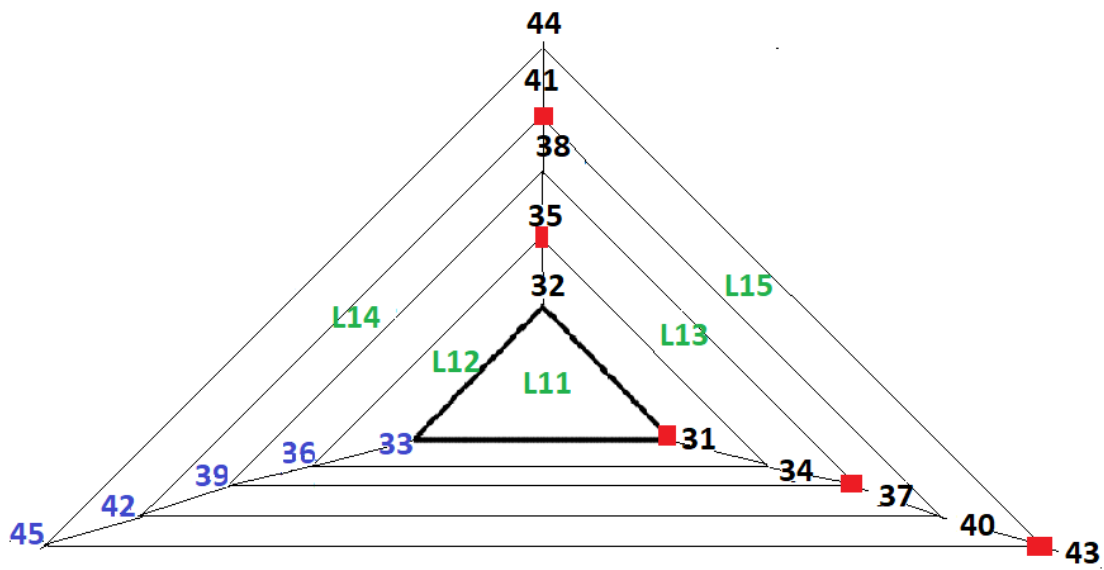

Figure 6. Sub Pyramid distribution for only numbers between 31 and 45

\subsection{Simpler Way to Check if a Natural Number Is a Prime Number}

Based on the pyramid distribution described in this paper. We conclude a simpler way to check if the number is a prime number or not in the cost of $<=\mathrm{O}(7)$.

If we need to check if the number $\mathrm{X}$ is prime or not?

1- Calculate X+1

2- Calculate X-1 
3- Calculate $\mathrm{X} / 3$

4- Calculate $(X+1) / 3$

5- Calculate $(\mathrm{X}-1) / 3$

6- Calculations in points 3 to 5 will produce only one number of $(X, X+1, X-1)$ will be divisible by 3 , the other two will not. This number will be the pyramid level base number (B) and the result of dividing this base number (B) by 3 will be our Level number (L) on the pyramid.

7- Based on the level number (even or odd number), and the operation applied on $\mathrm{B}(+1$ or -1 or +2$)$. The prime number will be located to the right of $\mathrm{B}$ or the top of $\mathrm{B}$.

Algorithm 1: Check if a natural number is a Prime

Input: any natural number $\mathrm{X}$

Output: True or False ( $\mathrm{X}$ is a Prime number or not).

Function IsPrime $(\mathrm{X})$

$\mathbf{B} \leftarrow$ a natural number divisible by 3 and is labeled as the base for pyramid level.

If $X \bmod 3==0$ then

$\mathrm{B}=\mathrm{X}$

else

$$
\mathrm{B}=[3-(\mathrm{X} \bmod 3)]+\mathrm{X}
$$

Pyramid Level $=\mathrm{B} / 3$

If IsComposite $(\mathrm{X})$ then $/ *$ exclude composite primes*/

Return False

If Pyramid Level mod $2==0$ and $(B+1==X$ or $B-1==X)$ then

Return True

If Pyramid Level mod $2==1$ and $(B+2==X$ or $B-2==X)$ then

Return True

Return False

End

For Example (1): $\mathrm{X}=131$ is a prime number?

Call Function IsPrime (131) defined in Algorithm 1

$1-131 \bmod 3=2$

2- $\quad \mathrm{B}=(3-2)+131=132$

3- Pyramid Level $=B / 3=44$

4- Pyramid level $\bmod 2=44 \bmod 2=0 \rightarrow$ pyramid level is an even Level $\rightarrow \quad$ Prime number should be $\mathrm{B}+1$ or B-1

5- Check if $\mathrm{X}=\mathrm{B}+1$ or $\mathrm{X}=\mathrm{B}-1$ :

$$
132-1=131=\mathrm{X} \text { then } \mathrm{X} \text { is Prime Number }
$$

Return True.

$\mathrm{X}=131$ is prime number.

Example (2): $X=1223$ is a prime number?

Call Function IsPrime (1223) defined in Algorithm 1

$1-1223 \bmod 3=2$ 
2- $\quad \mathrm{B}=(3-2)+1223=1224$

3- Pyramid Level $=\mathrm{B} / 3=408$

4- Pyramid level mod $2=408 \bmod 2=0 \rightarrow$ pyramid level is an even Level $\rightarrow$ Prime number should be B +1 or B-1

5- Check if $\mathrm{X}=\mathrm{B}+1$ or $\mathrm{X}=\mathrm{B}-1$ :

$1224-1=1223=\mathrm{X}$ then $\mathrm{X}$ is Prime Number

Return True.

$\mathrm{X}=1223$ is prime number.

Example (3), $X=380$ is a prime number?

Call Function IsPrime (380)

$1-380 \bmod 3=2$

2- $\quad \mathrm{B}=(3-2)+380=381$

3- Pyramid Level $=\mathrm{B} / 3=127$

4- Pyramid level mod $2=127 \bmod 2=1 \rightarrow$ pyramid level is an odd Level $\rightarrow \quad$ Prime number should be B -2 or $\mathrm{B}+2$

5- Check if $\mathrm{X}=\mathrm{B}+2$ or $\mathrm{X}=\mathrm{B}-2$ :

$381-2=379\langle>X$ and $381+2\langle>X$ then $X$ is not Prime Number

Return False.

$\mathrm{X}=380$ is NOT prime number.

Example (4), $\mathrm{X}=349$ is a prime number?

Call Function IsPrime(349)

$1-349 \bmod 3=1$

2- $\quad \mathrm{B}=(3-1)+349=351$

3- $\quad$ Pyramid Level $=\mathrm{B} / 3=117$

4- Pyramid level mod $2=117 \bmod 2=1 \rightarrow$ pyramid level is an odd Level $\rightarrow \quad$ Prime number should be $\mathrm{B}+2$ or B-2

5- Check if $\mathrm{X}=\mathrm{B}+2$ or $\mathrm{X}=\mathrm{B}-2$ :

$351-2=349=\mathrm{X}$ then $\mathrm{X}$ is Prime Number

Return True.

$\mathrm{X}=349$ is prime number.

Example (5), $\mathrm{X}=3491$ is a prime number?

Call Function IsPrime (3491)

$1-3491 \bmod 3=2$

2- $\quad \mathrm{B}=(3-2)+3491=3492$

3- $\quad$ Pyramid Level $=\mathrm{B} / 3=1164$

4- Pyramid level $\bmod 2=1164 \bmod 2=0 \rightarrow$ pyramid level is an even Level $\rightarrow$ Prime number should be B -1 or $\mathrm{B}+1$

5- Check if $\mathrm{X}=\mathrm{B}+1$ or $\mathrm{X}=\mathrm{B}-1$ :

$$
3492-1=3491=\mathrm{X} \text { then } \mathrm{X} \text { is Prime Number }
$$

Return True. 
$\mathrm{X}=3491$ is prime number.

\section{Distribution of Natural Numbers}

\subsection{Base Branch Distribution}

The Base branch is the main branch in the Pyramid. This branch will include all Natural numbers that are divisible by 3 and the result is an integer. The difference between numbers in each row is 3 .

Table 1. Base Branch

\begin{tabular}{|l|c|c|c|}
\hline ID & $\begin{array}{c}\text { Natural } \\
\text { Number }\end{array}$ & $\begin{array}{c}\text { Pyramid Layer } \\
\text { (Number / 3) }\end{array}$ & Composite Prime \\
\hline 1 & 3 & 1 & 0 \\
\hline 2 & 6 & 2 & 1 \\
\hline 3 & 9 & 3 & 1 \\
\hline 4 & 12 & 4 & 1 \\
\hline 5 & 15 & 5 & 1 \\
\hline.. &.. &.. & 1 \\
\hline 9999 & 9999 & 3333 & 1 \\
\hline
\end{tabular}

Table 1., includes all natural Numbers such that $(\mathrm{N} \bmod 3=0)$. The only prime number in this branch will be number 3 .

\subsection{Top Branch Distribution}

The Top branch is a branch in the Pyramid that will be on the top of the Base branch and will include all-natural numbers within $(-1$ or +1$)$ distance from the Base Branch.

This Branch will include all Natural Numbers that can be generated using Number 5 .

1- Any Natural number N, is located at ID $=([\mathrm{N}+1] / 6)-1$

2- Any Location ID is the location of a Natural number $\mathrm{N}=(5 *(\mathrm{ID}+1))+\mathrm{ID})$

3- All Natural numbers in Right Branch, that are divisible by a number $\mathrm{N}$ in this branch, are at Positions [Start; End; Step] = [ ID + N; Length of Right Branch; N].

For Example: -17 at position ID $=2$ the next number divisible by 17 will be at ID $=17+2=19$. And next number divisible by 17 will be at ID $=19+17$ and so on.

Table 2. Top Branch

\begin{tabular}{|l|c|c|c|c|c|}
\hline ID & $\begin{array}{c}\text { Natural } \\
\text { Number }\end{array}$ & $\begin{array}{c}\text { Pyramid Layer } \\
(\mathrm{ID} * 2)+2\end{array}$ & $\begin{array}{c}\text { Top Branch Formula } \\
\mathrm{N}=(5 *[\mathrm{ID}+1])+\mathrm{ID}\end{array}$ & $\begin{array}{c}\text { Base } \\
\mathrm{B}=(\text { Pyramid Layer } * 3)\end{array}$ & $\begin{array}{c}\text { Composite } \\
\text { Prime }\end{array}$ \\
\hline 0 & 5 & 2 & $(5 * 1)+0$ & 6 & 0 \\
\hline 1 & 11 & 4 & $(5 * 2)+1$ & 12 & 0 \\
\hline 2 & 17 & 6 & $(5 * 3)+2$ & 24 & 0 \\
\hline 3 & 23 & 8 & $(5 * 4)+3$ & 30 & 0 \\
\hline 4 & 29 & 10 & $(5 * 5)+4$ & 36 & 1 \\
\hline 5 & 35 & 12 & $(5 * 6)+5$ & &.. \\
\hline.. &.. &.. & $\ldots$. & & 0 \\
\hline
\end{tabular}

In Table 2. Each Natural Number in each row will be (+6) from the natural number in the previous row.

\subsection{Right Branch Distribution}

The Right branch is a branch in the Pyramid that will be on the right of the Base branch and will include all-natural numbers within $(-2$ or +2$)$ distance from the Base Branch.

This Branch will include all Natural Numbers that can be generated using Number 7 . 
Table 3. Right Branch

\begin{tabular}{|l|c|c|c|c|c|}
\hline ID & $\begin{array}{c}\text { Natural } \\
\text { Number }\end{array}$ & $\begin{array}{c}\text { Pyramid Layer } \\
(\mathrm{ID} * 2)+3\end{array}$ & $\begin{array}{c}\text { Top Branch Formula } \\
\mathrm{N}=(7 *[\mathrm{ID}+1])-\mathrm{ID}\end{array}$ & $\begin{array}{c}\text { Base } \\
\mathrm{B}=(\text { Pyramid Layer * 3) }\end{array}$ & $\begin{array}{c}\text { Composite } \\
\text { Prime }\end{array}$ \\
\hline 0 & 7 & 3 & $(7 * 1)-0$ & 9 & 0 \\
\hline 1 & 13 & 5 & $(7 * 2)-1$ & 15 & 0 \\
\hline 2 & 19 & 7 & $(7 * 3)-2$ & 21 & 0 \\
\hline 3 & 25 & 9 & $(7 * 4)-3$ & 33 & 0 \\
\hline 4 & 31 & 11 & $(7 * 5)-4$ & 39 & 0 \\
\hline 5 & 37 & 13 & $(7 * 6)-5$ & &.. \\
\hline.. &.. &.. & $\ldots$. & & \\
\hline
\end{tabular}

In Table 3. Each Natural Number in each row will be (+6) from the natural number in the previous row.

1- Any Natural number $\mathrm{N}$ in Right branch. will be divisible by 11 if we subtract 1 and multiply $\mathrm{N}$ by 5.5 . $([\mathrm{N}-1] * 5.5) \bmod 11=0$ for all $\mathrm{N}$.

2- Any Natural number $\mathrm{N}$ in Right branch. will be divisible by 5 if we add 1 and multiply $\mathrm{N}$ by 7.5 . ([N+1] * 7.5) $\bmod 5=0$ for all $\mathrm{N}$.

3- Any Natural number $\mathrm{N}$ is located at ID $=([\mathrm{N}-1] / 6)-1$.

4- Any Location ID is the location of a Natural number $\mathrm{N}=(7 *(\mathrm{ID}+1))-$ ID).

5- All Natural numbers in Right branch, that are divisible by a number $\mathrm{N}$ in this branch, are at Positions [Start; End; Step $]=[$ ID + N + Pyramid Layer at ID = 0; Length of Right branch; N].

For Example: -13 at position ID $=1$ the next number divisible by 13 will be at $\mathrm{ID}=13+1+3=17$. And next number divisible by 13 will be at ID $=17+13$ and so on.

Based on this distribution, to get a List of Primes numbers and composite Primes form a natural number space.

1- Exclude all even natural numbers, this will shrink the size of your space by $50 \%$.

2- Exclude all natural numbers that are divisible by 3, except number 3, This will shrink your space size by $30 \%$.

3- Use Top branch and Right branch distributions (in Table 2. And Table 3.) to shrink your space size by $15 \%$.

4- The final Prime number space should be in the range of $10 \%$ of natural number Space.

\section{Results}

There is a clear distribution in the pyramid representation for the Prime numbers which led us to get a simpler way to get the prime numbers in a subset of the numbers. And a simpler calculation algorithm to check any number if it is a prime number or not in a cost less than or equal to $\mathrm{O}(7)$.

\section{References}

Ares, S., \& Castro, M. (2006). Hidden structure in the randomness of the prime number sequence?. Physica A: Statistical Mechanics and its Applications, 360(2), 285-296. https://arxiv.org/abs/cond-mat/0310148v2

\section{Copyrights}

Copyright for this article is retained by the author(s), with first publication rights granted to the journal.

This is an open-access article distributed under the terms and conditions of the Creative Commons Attribution license (http://creativecommons.org/licenses/by/4.0/). 